\title{
Design of Remote Quiet Zones Using Spot-Type Sound Reducers
}

Article in Acta Acustica united with Acustica · September 2017

DOI: 10.3813/AAA.919113

CITATIONS

0

3 authors, including:

\section{Romain Boulandet}

École Polytechnique Fédérale de Lausanne 50 PUBLICATIONS 130 CITATIONS

SEE PROFILE
READS

9

Some of the authors of this publication are also working on these related projects:

Project
INTERACTS: Intelligent low-frequeNcy acousTic Equalization of Rooms using Active ConTrol Subwoofers View project

Hearing aids signal processing View project
Hervé Lissek

École Polytechnique Fédérale de Lausanne 139 PUBLICATIONS 342 CITATIONS

SEE PROFILE 


\title{
Design of remote quiet zones using spot-type sound reducers*
}

\author{
Romain Boulandet ${ }^{1)}$, Thomas Laurence ${ }^{1)}$, Hervé Lissek ${ }^{1)}$ \\ 1) LTS2, Ecole Polytechnique Fédérale de Lausanne, \\ Station 11, CH-1015, Lausanne, Switzerland. romain.boulandet@epfl.ch
}

\section{Summary}

This paper presents a local control approach to generate remote quiet zones. To deal with situations where global control can hardly be achieved, it is proposed to use an arrangement of spot-type sound reducers as originally suggested by Olson and May. Assuming that cross-coupling between control units is weak, each can be controlled independently and a decentralised feedback controller is implemented without the need for direct monitoring of the primary source. Active noise attenuation in the remote target region is achieved using a linear quadratic optimization based on prior knowledge of the transfer path of the system. The performance of a particular configuration comprising three control units is examined by numerical simulation and experimentally evaluated for a tonal noise source in a free-field environment. An average noise reduction of about $6 \mathrm{~dB}$ was measured in a target region of volume $0.25 \times 0.25 \times 0.25 \mathrm{~m}^{3}$ for a $160 \mathrm{~Hz}$ tonal primary source distant more than one wavelength from the secondary sources. The performance of the control system in relation to changes in the primary field is also considered with a view to extending the concept to more realistic enclosed sound field conditions in future work.

\section{Introduction}

In many workspaces and indoor public places, the low-frequency noise (LFN) of industrial machinery, electric transformer and air flow machinery including compressors or heating, ventilation and airconditioning (HVAC) systems is problematic. Epidemiological research showed an association between exposure to LFN sources and various adverse health effects such as annoyance, sleep-related problems, or concentrations difficulties. Unlike mid- and highfrequency noise, LFN is less attenuated by walls and other soundproofing structures due to the long wavelength involved, it masks higher frequencies more than it is masked by them, and ear protection devices are less effective against it. For low modal densities in the enclosure, a significant reduction in acoustic potential

\footnotetext{
${ }^{*}$ The archived file is not the final published version of the article
}

energy at the natural frequencies of the room can be achieved using remote secondary sources thanks to strong coupling with the primary field [1]. When the coupling between the sources is weak such as in a free space or for high modal densities, global control can hardly be achieved if the secondary sources are distant by more than half a wavelength from the primary source 2. In the latter cases, it is more efficient to design a control system that attempts to reduce noise only on a particular region of space, which is commonly referred to as a quiet zone. It is therefore of great interest to investigate the application of a local control whenever the target region is relatively small with respect to the wavelength of the primary excitation.

A quiet zone is generally described as a particular area of a sound field in which the sound pressure level is reduced by about $10 \mathrm{~dB}$ relative to the primary disturbance 3]. In a practical local noise control system, a secondary source is used to cancel the pressure at a closely spaced error microphone. The spatial extent around the minimization point is typically of the order of one tenth of a wavelength at the excitation frequency. The effect of spacing between the primary and secondary sources on the control performance was studied by Joseph et al. 4. It was found that the secondary source should not be too far away in order not to increase the sound pressure level elsewhere in space. The effect of moving the error microphone away from the secondary source was studied numerically by David and Elliott [5]. It was observed that the spatial extent of the quiet zone can be increased up to a limiting value of about one tenth of a wavelength both in a uniform and a diffuse primary field. As shown by Elliott and Garcia-Bonito [6, the size of the quiet zone can be further increased by minimizing both the pressure and pressure gradient, but this requires two independent secondary sources. The design of larger quiet zones in a free space was also examined by Guo et al. [8, 9] using a multi-input and multioutput controller. They showed that there exists a range of optimal spacing for the control loudspeakers and error microphones which also minimizes the total power output outside the quiet zone. Local control in diffuse sound field was explored by Tseng [10], where a convex, nonlinear constrained optimization problem was solved to design a secondary field that maximizes 
the area of the $10 \mathrm{~dB}$ quiet zone instead of minimizing the sum of the squared pressures.

When a sound field is temporarilly deterministic and a reference signal properly correlated to the primary disturbance is available, the quiet zone is typically implemented by a feedforward control system [8]. In many practical applications, however, the disturbance is not tonal but rather broadband and may originate from several distinct noise sources. In this case, a reference signal is generally not available and feedback control should be considered instead. This type of control architecture is generally less stable than feedforward control and careful examination of the closed loop system is necessary to ensure stability [11, 12, 13.

The idea of using a loudspeaker and microphone nearby in a feedback loop was originally proposed by Olson and May in the early 1950s [14, 15]. In their pioneering work, the authors suggested two distinct modes of operation. In the "electronic sound absorber" principle, a loudspeaker is adjusted using a microphone in a negative feedback loop to maximize its own acoustic power absorption. This control approach is particularly suitable for achieving active acoustic absorption in cavities when the standing waves are an obvious problem [2, 16]. Based on this principle, an active equivalent of the quarter-wave resonator can be achieved by pressure minimization on the rear face of a thin porous layer having a flow resistance equal to the characteristic impedance of air [17, 18. More recently, Rivet et al. showed that a collocated loudspeaker-microphone pair, both being connected by a model-based transfer function, can be used for matching the diaphragm impedance to a target specific acoustic impedance, which has the effect of damping the standing waves in the enclosure [19. In the "spot-type sound reducer" principle, however, the strength of the secondary source is adjusted from the microphone to produce a zone of quiet nearby. In [15] for instance, it is suggested to use this system as a low-frequency noise reducer in factories, shops and offices where the noise level is high and the position of the person is fixed.

In many practical applications, it is not desirable to place the error sensor(s) in the region of desired acoustic attenuation. As reviewed by Moreau et al. [20, there has been a considerable interest over the past decades in monitoring the sound field within the region of control with sensors outside this region. In the early 1990s, Elliott and David proposed using a virtual microphone arrangement that can move the zone of quiet so that it is further away from the secondary sources than the physical error microphone [21. This arrangement is based on the assumption that, at low frequencies, the spatial rate of change of the primary sound field is small at the physical and at the virtual microphone locations [7. In the early 2000s, Kestell et al. showed that the zone of quiet could be further ex- tended using a virtual energy density sensor from the pressure and pressure gradient [22], considering that the weighted summation of pressure and velocity is much more spatially uniform than pressure only. It should also be noted that local control using phasedarray sensors was also suggested to achieve directionally sensitive detection through acoustic beam steering techniques [23], thereby producing acoustic beams which intersect to define a quiet area far away from the sensors.

In this article, we present a decentralised control system for remote quiet zone design inspired by Olson and May's "spot-type sound reducer" concept, which does not require direct monitoring of the primary field nor use sensor in the target region. The remainder of the paper is organized as follow. The design of the multichannel feedback controller is addressed in Section 2 where a linear quadratic optimization problem is solved to derive the feedback gains. The performance of a particular configuration comprising three collocated loudspeaker and microphone pairs is evaluated in Section 3 assuming a tonal primary source in a free field environment. Measurements are compared to simulations in the case of a single harmonic primary source. To ensure that the system can be implemented in the presence of more complex sound fields, the performance is also investigated experimentally for multiple coherent sound sources distributed randomly around the target region. The benefits and limitations of this concept to generate remote quiet zones in enclosed sound fields are then discussed.

\section{Problem formulation}

This section presents the theoretical framework formulation for the design of a control system to generate a remote quiet zone. The main goal is to develop a decentralised controller that can operate without direct monitoring of the primary source and without error sensors in the target region. A two-step approach is proposed: 1) a linear quadratic optimization problem is solved to calculate the optimal control input that minimizes the sum of the squared pressures in the target region, and 2) the optimal solution is reformulated as a complex gain matrix using pre-determined transfer functions relating the target region and the control units, allowing for a decentralised control approach.

\subsection{Control system design}

An illustration of the physical arrangement considered in this study is shown in Fig. 11, where a set of $n$ tightly coupled source-sensor pairs but lightly cross coupled control units is used to minimize the disturbance generated by an unknown primary source at $m$ points defining the remote target region. Let us first examine the open-loop control system as shown in 

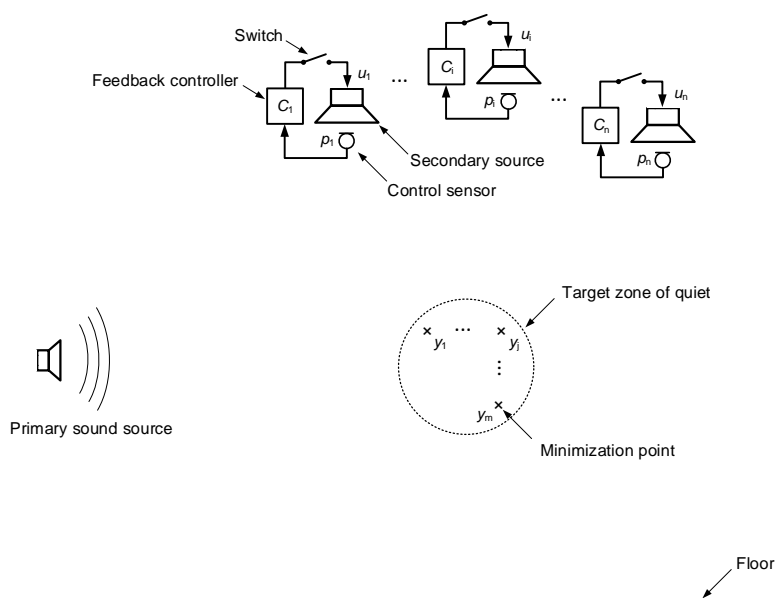

Figure 1: Illustration of the multi-channel control arrangement assumed to create a remote zone of quiet.

Fig. 2a. Assuming linearity and superposition principle, the system response at the $m$ minimization points can be written in matrix form as

$$
\mathbf{y}=\mathbf{G u}+\mathbf{d}
$$

where $\mathbf{y}$ is the $m \times 1$ vector of the total complex pressure, $\mathbf{u}$ is the $n \times 1$ vector of control inputs, $\mathbf{G}$ is the $m \times n$ matrix of transfer functions relating the control inputs and the minimization points, and $\mathbf{d}$ is the $m \times 1$ vector of the disturbance.

The cost function that minimizes the sum of the squared complex pressures with a uniform, quadratic control effort weighting [27] can be written as

$$
J=\mathbf{y}^{H} \mathbf{y}+\beta \mathbf{u}^{H} \mathbf{u}
$$

where the superscript $H$ is the complex conjugate of the vector transpose. The term on the right-hand side of Eq. (2), which is proportional to the control effort, is used to make the stability and performance of the controller robust to uncertainties in the modelling of the system or changes over time. Substituting Eq. (1) into Eq. (2), and after some further manipulations, the cost function can be rewritten in the Hermitian quadratic form 2

$$
J=\mathbf{u}^{H}\left(\mathbf{G}^{H} \mathbf{G}+\beta \mathbf{I}\right) \mathbf{u}+\mathbf{u}^{H} \mathbf{G}^{H} \mathbf{d}+\mathbf{d}^{H} \mathbf{G u}+\mathbf{d}^{H} \mathbf{d}
$$

where $\mathbf{I}$ is the identity matrix. For a feedback control system with an equal number of collocated actuators and sensors, it can be shown that the cost function (3) has a unique global minimum, provided that $\mathbf{G}^{H} \mathbf{G}+\beta \mathbf{I}$ is positive definite 2. The optimal open-loop control input can therefore be derived as

$$
\mathbf{u}_{o p t}=-\left[\mathbf{G}^{H} \mathbf{G}+\beta \mathbf{I}\right]^{-1} \mathbf{G}^{H} \mathbf{d}
$$

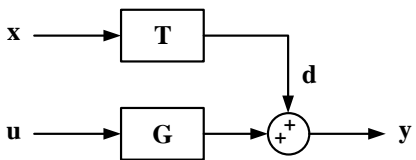

(a)

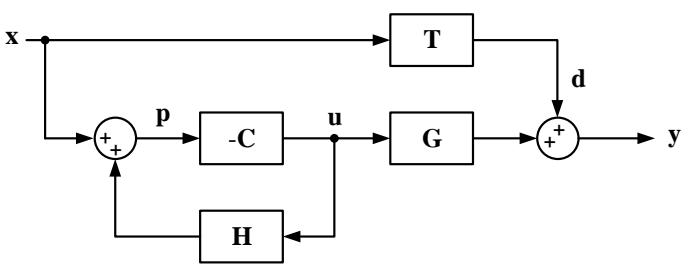

(b)

Figure 2: Block diagram of the open loop feedforward (a) and feedback (b) control system designed to reduce noise in a remote target region.

Substituting the expression for $\mathbf{u}_{o p t}$ into Eq. (3), the minimum value of $J$ is found to be

$$
J_{\min }=\mathbf{d}^{H} \mathbf{d}-\mathbf{d}^{H} \mathbf{G}\left[\mathbf{G}^{H} \mathbf{G}+\beta \mathbf{I}\right]^{-1} \mathbf{G}^{H} \mathbf{d}
$$

and the maximum achievable attenuation (in $\mathrm{dB}$ ) can be derived from Eqs. (3) and (5) as

$$
\Delta J=10 \log _{10}\left(J(\mathbf{u}=0) / J_{\min }\right)
$$

As indicated in Eq. (4), the optimal control input is calculated for a given primary disturbance $\mathbf{d}=\mathbf{T} \mathbf{x}$. In a typical feedforward control scheme, $\mathbf{T}$ would be the transfer path between the minimization points and the reference signal taken from the primary source. A prior model or measurement of $\mathbf{T}$ can hardly be achieved if the primary disturbance is due to a large number of incoherent processes, or if the reference signal is not available. Moreover, any change in the primary disturbance can make the control system not optimal anymore since the control variable adjustment is not error-based. In the proposed control scheme, $\mathbf{T}$ is the $m \times n$ matrix of transfer functions between the incident pressure at the control points and the incident pressure at the minimization points; $\mathbf{x}$ is therefore the $n \times 1$ vector of the incident pressure at the control points generated by the primary source alone. In what follows, we present the process of transferring from the centralised feedforward control problem discussed above into an decentralised feedback formulation where the control variables also depend on the near field pressure of the secondary sources.

\subsection{Feedback controller}

Let us consider now the multichannel control arrangement illustrated in Fig. 1 with all the switches closed. 
Figure $2 \mathrm{~b}$ shows the corresponding closed loop system, the response of which is written as

$$
\mathbf{p}=\mathbf{H u}+\mathbf{x}
$$

where $\mathbf{p}$ is the $n \times 1$ vector of total complex pressures at the control sensors output and $\mathbf{H}$ is the $n \times n$ matrix of transfer functions between the control inputs and the control sensors outputs. The feedback control law can be formulated as

$$
\mathbf{u}=-\mathbf{C p}
$$

where $\mathbf{C}$ is the $n \times n$ matrix of the feedback gains. In the proposed decentralised approach, the control gains in each feedback loop are defined as the ratio between the optimal control inputs and the resulting local pressure, which can be expressed for a stationary harmonic disturbance at frequency $\omega_{0}$ as

$$
-\mathbf{C}\left(\mathrm{j} \omega_{0}\right):=\operatorname{diag}\left(-\frac{u_{o p t, 1}}{p_{1}},-\frac{u_{o p t, 2}}{p_{2}}, \cdots,-\frac{u_{o p t, n}}{p_{n}}\right)
$$

Substituting now Eq. (4) into Eq. (7), the total complex pressure at the $i$ th control sensor can be expressed in terms of the summation for the $i$ th element of $\mathbf{p}$ as

$$
p_{i}=x_{i}-\sum_{j=1}^{n} \sum_{k=1}^{n} H_{i k} W_{k j} x_{j}
$$

where $\mathbf{W}=\left[\mathbf{G}^{H} \mathbf{G}+\beta \mathbf{I}\right]^{-1} \mathbf{G}^{H} \mathbf{T}$. Similarly, Eq. (4) can be rewritten in terms of the summation for the $i$ th element of $\mathbf{u}_{\text {opt }}$ as

$$
u_{o p t, i}=\sum_{j=1}^{n} W_{i j} x_{j}
$$

The optimal gain (9) to be applied between the $i$ th control sensor output and the $i$ th control actuator input at a given frequency, can therefore be derived from Eqs. (10) and (11) as

$$
C_{i}=-\frac{\sum_{j=1}^{n} W_{i j} x_{j}}{x_{i}+\sum_{j=1}^{n} \sum_{k=1}^{n} H_{i k} W_{k j} x_{j}}
$$

Assuming now that each element in $\mathbf{x}$ can be expressed as a function of a reference signal $r$ so that $\mathbf{x}=\mathbf{R} r$, Eq. 12 can be rewritten as

$$
C_{i}=-\frac{\sum_{j=1}^{n} W_{i j} R_{j}}{R_{i}+\sum_{j=1}^{n} \sum_{k=1}^{n} H_{i k} W_{k j} R_{j}}
$$

Equation 13 gives the expression of the complex optimal gain to be applied to the $i$ th control unit in order to minimize the sum of the squared pressures in the target region. As shown in Eq. (13), direct monitoring of the primary source is no longer required if the signal $r$ is taken from the output of one of the control sensors. Only prior knowledge of the acoustic transfer path $\mathbf{T}$ between control points and minimization points is necessary. As will be seen in Section 3, $\mathbf{G}$ and $\mathbf{H}$ are identified during an off-line design step by activating each secondary source independently, while $\mathbf{T}$ and $\mathbf{R}$ are obtained with the help of an additional loudspeaker used as primary source.

\subsection{Stability consideration}

The stability of a general multichannel control system can be determined by examining whether the locus of the real and imaginary parts of eigenvalues of $\mathbf{H}(\mathrm{j} \omega) \mathbf{C}(\mathrm{j} \omega)$ encloses the Nyquist point $(-1,0)$ as $\omega$ varies from $-\infty$ to $\infty$ [24]. For the control configuration under consideration, $\mathbf{H}$ is a strictly positive real matrix, i.e. $\mathbf{H}^{H}(\mathrm{j} \omega)+\mathbf{H}(\mathrm{j} \omega)>0$ for all $\omega \in \mathbb{R}$, provided that dual and collocated source-sensor pairs are used. In a classical decentralised feedback control problem where the control objective is disturbance rejection for instance [25], $\mathbf{C}$ is a diagonal matrix which is assumed to have a positive gain $\gamma$ on each channel, so that $\mathbf{C}=\gamma \mathbf{I}$. Then, it can be shown that the feedback control system is unconditionally stable since the system and controller are both passive [26. When the actuator-sensor pairs are not collocated and dual, unconditional stability is not guaranteed at all frequencies. Moreover, if some of the feedback gains have negative real part, on the other hand, the closed loop system may be unstable. This problem is discussed below considering a complex envelope controller used to handle any complex gain while ensuring the stability of the multichannel control system.

\subsection{Complex envelope controller}

In the following we address the practical implementation of any complex gain derived from Eq. (13), including those with negative real part values. Figure 3 shows the block diagram of the complex envelope controller for the $i$ th collocated microphone-loudspeaker pair. As shown in Fig. 3, the equation of motion of the loudspeaker diaphragm is given by [19]

$$
S_{d} p_{i}=Z_{m}(\omega) v_{i}+B l i_{i}
$$

where $p_{i}$ and $v_{i}$ are respectively the total pressure at, and the velocity of, the $i$ th diaphragm, $i_{i}$ is the current flowing through the coil, $S_{d}$ is the radiating surface area of the diaphragm, $Z_{m}$ is the mechanical impedance of the loudspeaker, and $B l$ is the electrodynamic transduction coefficient. The total pressure at the $i$ th loudspeaker diaphragm can be expressed as

$$
p_{i}=Z_{r}(\omega) q_{i}+z_{i}
$$

where $Z_{r}$ is the acoustic radiation impedance, $q_{i}$ is the volume velocity generated by the loudspeaker and $z_{i}$ is the disturbance at the $i$ th pair caused by both the primary sound field and the other lightly coupled secondary sources. 


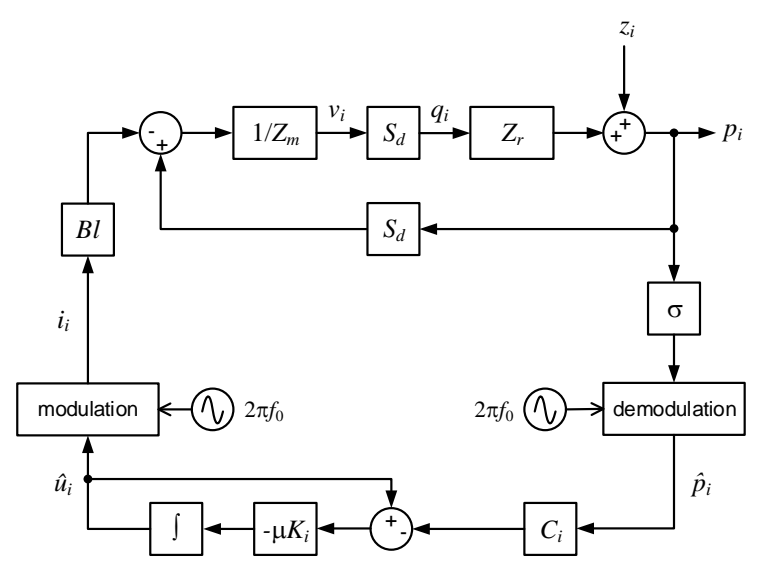

Figure 3: Block diagram of the complex envelope controller applied to the $i$ th microphone-loudspeaker pair.

In complex envelope representation, the current delivered at the output of the controller by modulation with $\hat{u}_{i}(t)$ can be written as

$$
i_{i}(t)=\operatorname{Re}\left[\hat{u}_{i}(t) \exp \left(\mathrm{j} 2 \pi f_{0} t\right)\right]
$$

where Re[.] denotes the real part and $\hat{\mathbf{u}}_{i}$ is the complex envelope of $i_{i}$ for the carrier frequency $f_{0}=$ $\omega_{0} / 2 \pi$. Similarly, the complex envelope of the total pressure at the $i$ th microphone can be obtained by demodulation of $p_{i}$ as

$$
\hat{p}_{i}(t)=2 g(t) \otimes\left(\sigma p_{i}(t) \exp \left(-\mathrm{j} 2 \pi f_{0} t\right)\right)
$$

where $\otimes$ denotes the convolution operator, $g$ is the impulse response of a low-pass filter of cut-off frequency $\omega_{c} \ll \omega_{0}$, and $\sigma$ is the microphone sensitivity (in $\mathrm{V} \mathrm{Pa}^{-1}$ ). As shown in Fig. 3, the harmonic controller used to minimize the distance $\left|\hat{u}_{i}-C_{i} \hat{p}_{i}\right|$ is written as

$$
\frac{\mathrm{d} \hat{u}_{i}}{\mathrm{~d} t}=-\mu K_{i}\left[\hat{u}_{i}(t)+C_{i} \hat{p}_{i}(t)\right]
$$

where $\mu$ is a positive real gain and $K_{i}$ is a complexvalued gain.

Expressing now Eq. (7) using the complex envelope as $\hat{\mathbf{p}}=\mathbf{H} \hat{\mathbf{u}}+\hat{\mathbf{z}}$, and after some manipulations, the multichannel controller can be expressed from Eq. (18) as

$$
\frac{\mathrm{d} \hat{\mathbf{u}}}{\mathrm{d} t}=-\mu \mathbf{K}(\mathbf{I}+\mathbf{C H}) \hat{\mathbf{u}}+\mu \mathbf{K} \mathbf{C} \hat{\mathbf{z}}
$$

where $\mathbf{K}$ is the complex-valued compensation matrix. The closed-loop controller $\sqrt{19}$ is stable, provided that all the eigenvalues of $\mu \mathbf{K}(\mathbf{I}+\mathbf{C H})$ have positive real parts. Since $\mathbf{C}$ is a diagonal matrix, the algorithm (19) is centralised if $\mathbf{K}$ is a fully-populated matrix, and decentralised if $\mathbf{K}$ is diagonal. More details on the design of the complex envelop controller including the calculation of the diagonal compensation matrix $\mathbf{K}$ to implement a stable decentralised control can be found in [28, 29, 30.

\section{Results}

This section provides numerical and experimental results that show the performance of the control system described in Section 2 to create a quiet zone in a free field environment with pure tone excitation. Without losing generality, a particular configuration comprising three control units located above the target region is used below as an example.

\subsection{Numerical investigation}

Figure 4 illustrates the geometry of the control arrangement considered below. The acoustic domain is a hemisphere of radius of $3.5 \mathrm{~m}$. A sound hard boundary condition is used for the ground $(z=0)$ and nonreflecting boundary condition is applied to the remaining part using a perfect matched layer $(0.6 \mathrm{~m}$ thickness). The tonal primary sound field is generated by a point source located at $(x, y, z)=(-1.5,0,1.1)$ $\mathrm{m}$. The source strength is $0.001 \mathrm{~m}^{3} \mathrm{~s}^{-1}$. The secondary sources are modeled as 5 inches diameter pistons mounted in a circular reflective panel of $1.2 \mathrm{~m}$ radius, the center of which is located at $(x, y, z)=$ $(1.5,0,2) \mathrm{m}$. As can be seen in Fig. 4. the target re-

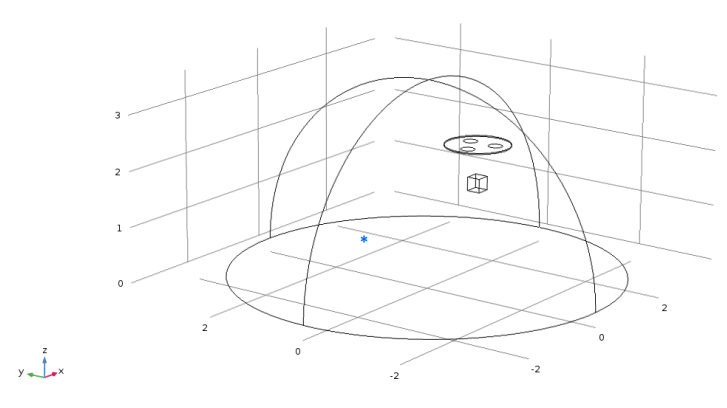

Figure 4: Geometry of the control arrangement used in the numerical investigation.

gion is defined by 8 minimization points distributed at the vertices of a cube $(0.25 \mathrm{~m}$ edge $)$. The center of the cube is located at $(x, y, z)=(1.5,0,1.3) \mathrm{m}$, i.e. at $3 \mathrm{~m}$ from the point source. The target volume of the quiet zone is therefore $0.25 \times 0.25 \times 0.25=15.610^{-3} \mathrm{~m}^{3}$. This geometry was implemented in the finite element simulation software COMSOL Multiphysics to provide the control algorithm with the transfer functions necessary to calculate the optimal complex gains as indicated in Eq. 13, and to compute the sound pressure levels (SPL) before and after control. The calculation of the optimal complex gains was performed in Matlab. 

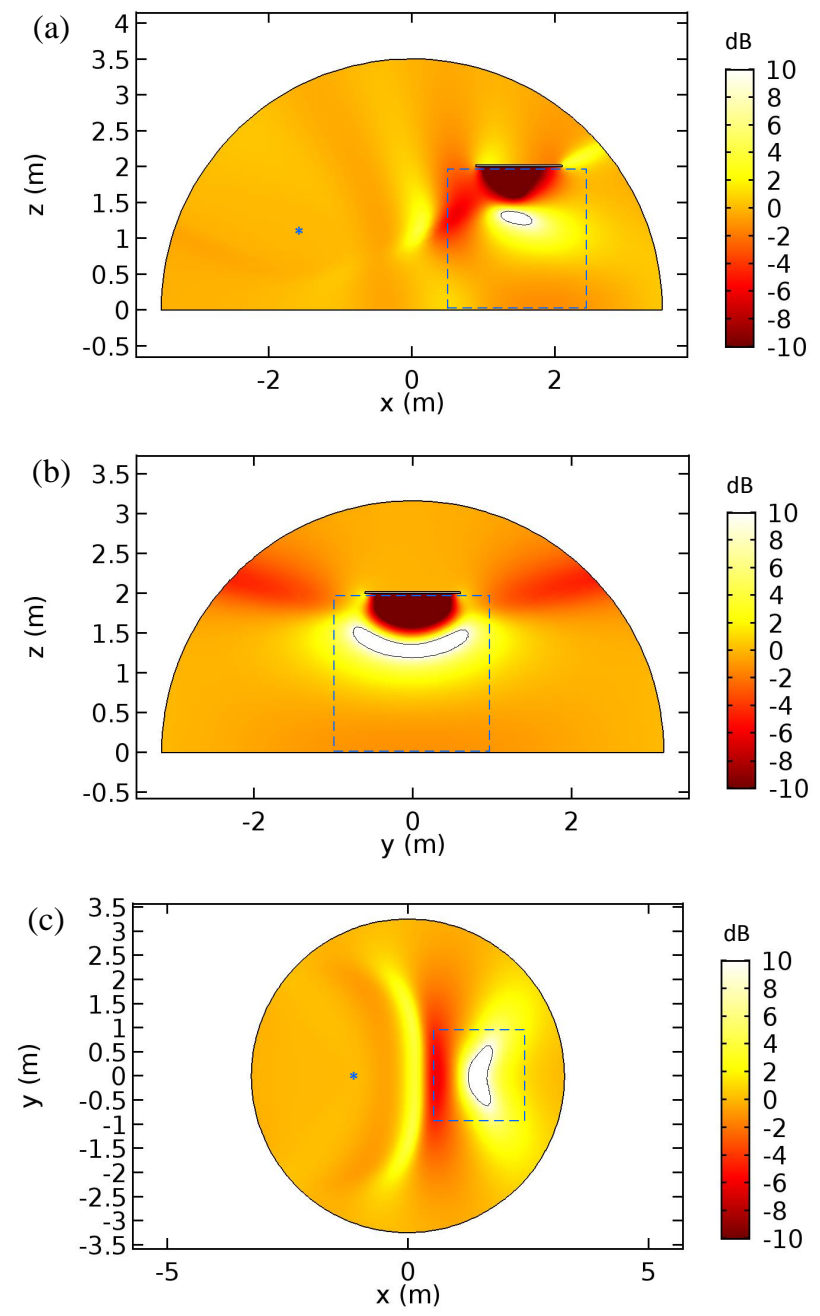

Figure 5: Change in sound pressure level computed for a single point source, where the marker * indicates the location of the primary source and the dashed blue line represents the $2 \times 2 \mathrm{~m}^{2}$ measurement grid that was used in the experiment discussed below; (a) cut view in the $x-z$ plane, (b) cut view in the $y-z$ plane, and (c) cut view in the $x-y$ plane.

Figure 5 shows the computed performance, quantified by the change in sound pressure level (SPL), where (a) is a cut view in the $x-z$ plane at $y=0$, (b) is a cut view in the $y-z$ plane at $x=1.5 \mathrm{~m}$, and (c) is a cut view in the $x-y$ plane at $z=1.3 \mathrm{~m}$, respectively. The frequency of excitation was chosen to be $160 \mathrm{~Hz}$ (corresponding to $\lambda \simeq 2.15 \mathrm{~m}$ ). In Fig. 5 . positive values in decibels represent sound reduction as a result of control, while negative values indicate an increase in sound pressure level. As can be seen in Fig. 5, an acoustic attenuation of more than $10 \mathrm{~dB}$ is achieved around the target region. Figure 5 shows that the secondary sources produce an increase of the sound pressure level in the near field of the loudspeakers and, to a lesser extent, around the quiet zone due to the constructive wave summation.
The following section presents simulation results which reproduce previous work on the diffraction effect of the listener's head modeled by a rigid sphere [7, and on the effect of the diaphragm surface area of the secondary sources on the size of the quiet zone 4. Figure 6 (left) illustrates the effect of a rigid diffraction sphere of $8.75 \mathrm{~cm}$ radius in the middle of the quiet zone. As can be seen in the left-hand side of Fig. 6] inserting the rigid sphere in the target region has little influence on the acoustic performance. A 0.3 $\mathrm{dB}$ decrease is obesrved with the feedback gains derived from the initial configuration when the diffraction sphere is placed in the desired quiet zone (see Tab. 1). Not shown in this paper, taking into account the diffraction sphere in the initialization stage leads to similar results. Figure 6 (right) shows the change in SPL when the radius of the secondary source is twice the one used in the initial configuration. As shown in the right-hand side of Fig. 6, increasing the radiating surface area of the secondary sources tends to increase the size of the quiet zone, as already observed by Joseph et al. (1994) in the case of a single secondary loudspeaker and a single point in its near field [4].

Table 1: Average reduction in sound pressure level computed in the quiet zone for the studied configurations.

\begin{tabular}{lcc}
\hline Configuration & Piston diameter & $\Delta L_{p}$ \\
\hline Initial & $5 \mathrm{in}$ & $5.9 \mathrm{~dB}$ \\
Rigid diffraction sphere & $5 \mathrm{in}$ & $5.6 \mathrm{~dB}$ \\
Larger radiating surface & $10 \mathrm{in}$ & $7.4 \mathrm{~dB}$ \\
\hline
\end{tabular}

Table 1 summarizes the computed performance in terms of SPL reduction for the initial control arrangement shown in Fig. 4, with an without accounting for a diffraction sphere in the middle of the target region, and when the radius of the secondary sources is increased. The acoustic attenuation $\Delta L_{p}$ in the quiet zone is obtained by averaging the sound pressure levels over all the minimization points. As can be seen in Tab. 1. the change in SPL is positive for the studied configurations, showing that the primary perturbation is reduced in the target region. However, it is found that the expected $10 \mathrm{~dB}$ acoustic attenuation is not reached at all the minimization points. This is because several minimization points distributed in space are considered. If a quiet zone is commonly defined as the region of space where a primary sound field can be reduced by $10 \mathrm{~dB}$, this usually involves a secondary source and a single minimization point. In this case, the spatial extent of the quiet zone is of the order of $\lambda / 10$. In this study, on the other hand, the solution is optimal but has no constraint on the homogeneity of the attenuation at the minimization points. As stated in Eq. (2), the cost function relates to the 

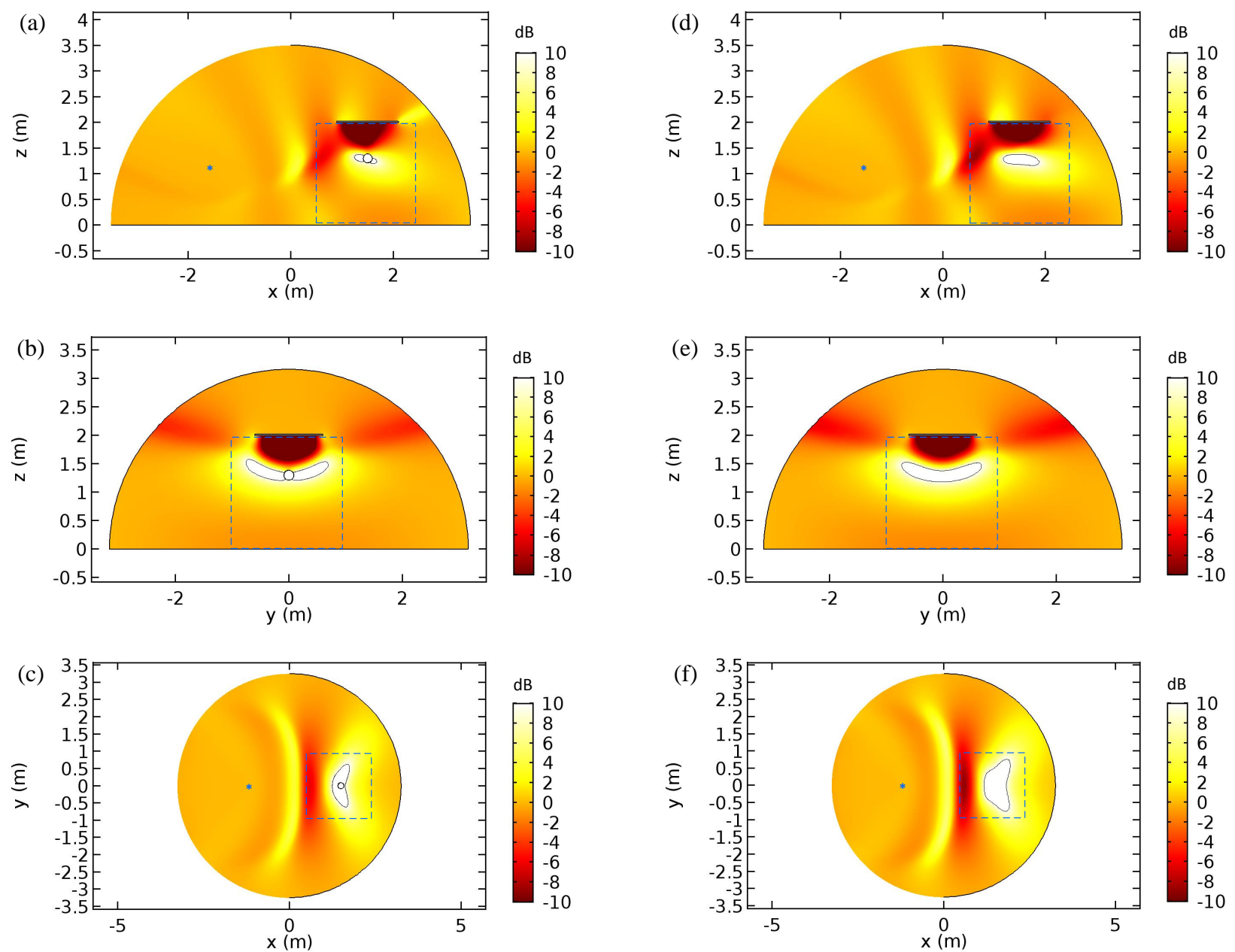

Figure 6: Change in sound pressure level computed for a single point source represented by marker * when a rigid sphere is inserted into the target region (left) and using secondary sources with larger piston area (right); (a) and (d) cut view in the $x-z$ plane, (b) and (e) cut view in the $y-z$ plane, and (c) and (f) cut view in the $x-y$ plane, respectively.

sum of the squared pressures and not the pressure at each point. Thus, using several spatially distributed minimization points, it can be seen that the zone of quiet can be extended in comparison with that expected for a single minimization point, as shown in Figs. 5 and 6 .

We now examine the effect of moving the primary source around the control arrangement with the optimal gains calculated for the initial configuration (see Fig. 5). Figure 7 shows a polar plot of the average change of sound pressure level in the quiet zone computed in steps of 10 degrees. As can be seen in Fig. 7, the performance of the control arrangement is sensitive to the azimuth of the primary source. An overall attenuation greater than $6 \mathrm{~dB}$ is obtained in the target region when the primary source is within 50 degrees and +50 degrees with respect to the initial configuration. On the other hand, when the primary source is located more than 80 degrees from the initial configuration, the average SPL in the quiet zone increases up to $3.5 \mathrm{~dB}$ when the primary source is diametrically opposed.

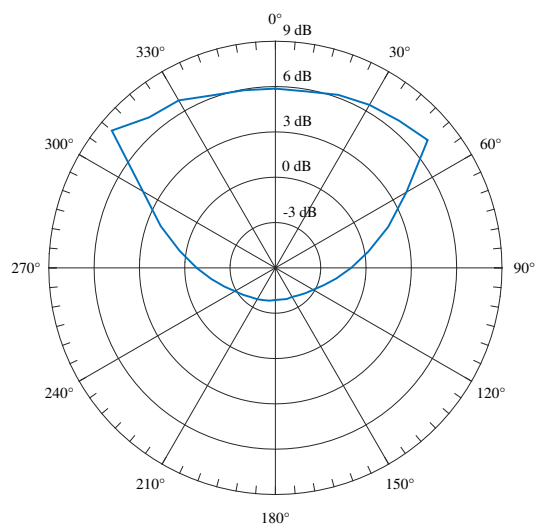

Figure 7: Polar plot in $\mathrm{dB}$ of spatially averaged sound pressure level change computed in the quiet zone. 


\subsection{Experimental setup}

In order to validate the numerical findings, experiments were carried out in an anechoic chamber (volume $200 \mathrm{~m}^{3}$, cut-off frequency $80 \mathrm{~Hz}$ ). Figure 8 shows a picture of the experimental configuration in which a large wooden panel is used as a floor and placed $2 \mathrm{~m}$ below the control units. The control arrangement is composed of three units each comprising a 6.5 inch Visaton W $170 \mathrm{~S}$ loudspeaker mounted in a $10 \mathrm{~L}$ closed-box and a $1 / 4$ inch model 130D20 ICP microphone located about $1 \mathrm{~cm}$ from the loudspeaker diaphragm. As in simulation, the loudspeakers are arranged on a wooden panel of $1.2 \mathrm{~m}$ in diameter and spaced at intervals of 120 degrees. The digital processing is implemented with a National Instruments real-time CompactRIO platform. The NI 9215 module (10 V 16-bit resolution) is used for data acquisition and the NI 9263 (10 V 16-bit resolution) is used for analog output. As depicted in Fig. 4, a set of 8 microphones distributed at the vertices of a cube of 0.25 $\mathrm{m}$ edge is used during the initialization phase to obtain the transfer functions necessary to implement the control algorithm. The center of this microphone array is approximately $1.3 \mathrm{~m}$ above the floor and $3.5 \mathrm{~m}$ from the loudspeaker used as a primary noise source. Note that the microphone array shown in Fig. 4 is only required during initialization to get the transfer paths $\mathbf{G}$ and $\mathbf{T}$ between the control units and the minimization points and is removed after.

The transfer functions between the input signal of each source and the sensors output were measured using a swept sine signal in the frequency range $30 \mathrm{~Hz}-300 \mathrm{~Hz}$, allowing identification of the matrices $\mathbf{G}, \mathbf{H}, \mathbf{R}$ and $\mathbf{T}$. The transfer functions between the input of the secondary sources and the output of the control microphones are illustrated in Fig. 9 as an example. As can be seen in Fig. 9, the gain of the offdiagonal terms in $\mathbf{H}$ is lower than $10 \mathrm{~dB}$ compared to

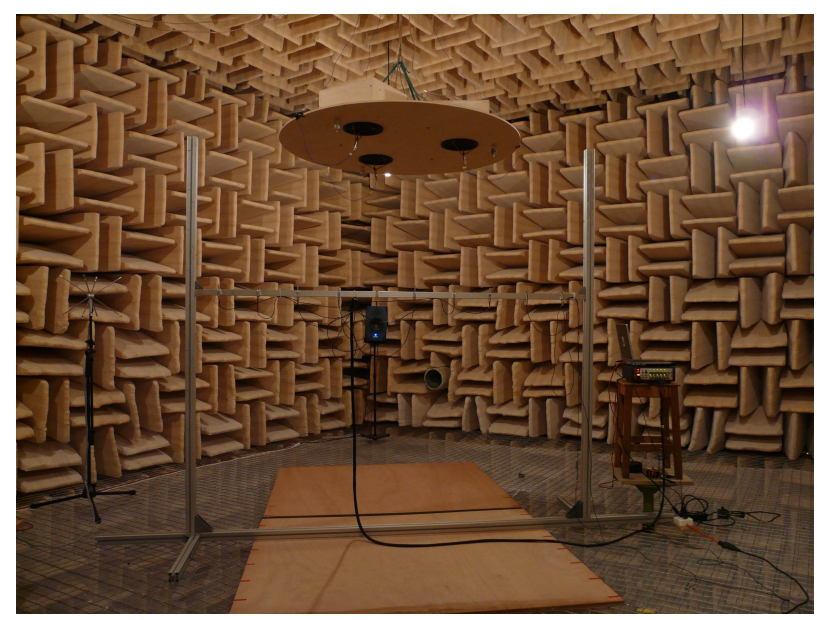

Figure 8: Picture of the experimental configuration during performance evaluation.
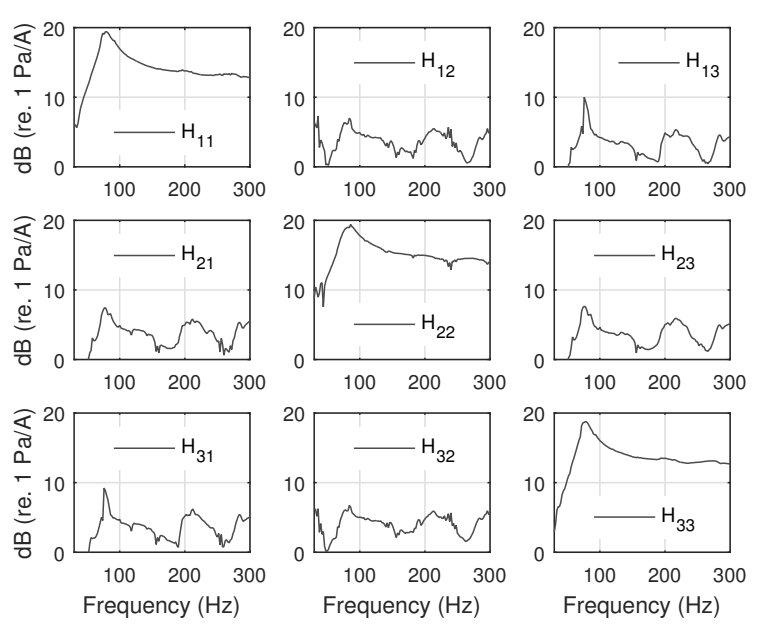

Figure 9: Frequency response of the measured transfer functions $\mathbf{H}$.

the diagonal terms, showing that cross-coupling between the control units is small.

Figure 10a shows the maximum achievable attenuation calculated from Eq. 13 for $\beta=0.1$ as a function of the frequency. As shown in Fig. 10 , the maximum sound reduction is expected in the frequency range 70 $\mathrm{Hz}-190 \mathrm{~Hz}$, i.e. above the resonance frequency of the closed-box loudspeaker used in this study. The lower system performance below $70 \mathrm{~Hz}$ is due to the low frequency response of the closed loudspeakers used as secondary sources. Moreover, the low attenuation expected between $190 \mathrm{~Hz}$ and $250 \mathrm{~Hz}$ is probably related to the cross coupling between the control units. As can be observed in Fig. 9, the off-diagonal terms in the matrix $\mathbf{H}$ of the control system shows a peak in this frequency range. Figure $10 \mathrm{p}$ shows the real part and imaginary part of the optimal gains calculated from Eq. 13) for $\beta=0.1$ as a function of the frequency. Above the resonance frequency of the loudspeaker, the frequency response of optimal gains is relatively smooth except in the frequency range 190 $\mathrm{Hz}-250 \mathrm{~Hz}$ where the maximum attainable attenuation is found to be the lowest, as shown in Fig. 10a.

\subsection{Experimental investigation}

This section presents experimental results measured with a tonal primary source in the anechoic chamber, as shown in Fig. 8. The acoustic performance of the control system is evaluated as the ratio in $\mathrm{dB}$ of the sum of the squared sound pressure measured over a surface area around the desired zone of quiet before and after control. A linear arrangement of 10 microphones spaced $0.2 \mathrm{~m}$ apart (see Fig. 8) was moved horizontally and vertically, allowing the change in sound pressure level to be observed over three orthogonal planes intersecting the center of the target 


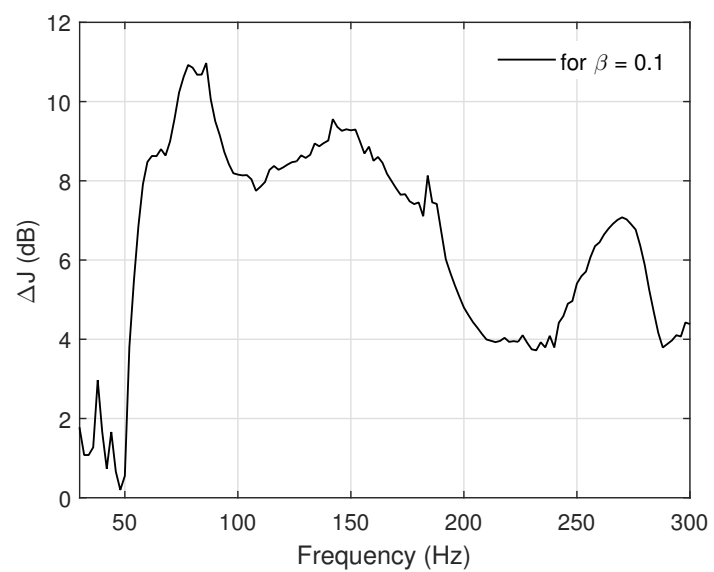

(a)
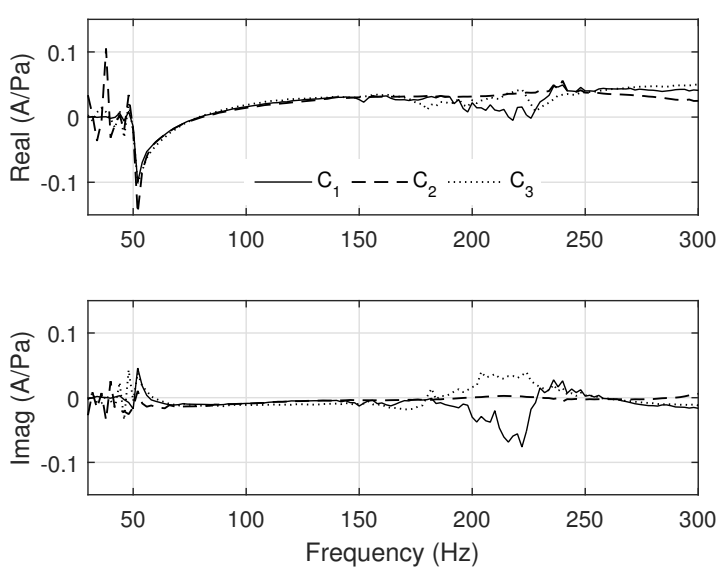

(b)

Figure 10: Maximum achievable attenuation (a) calculated from Eq. 60 and real part and imaginary part of the optimal gains (b) calculated from Eq. $\sqrt{13}$ in the frequency range $30-300 \mathrm{~Hz}$ for $\beta=0.1$, respectively.

region, as in the simulations. The measurement grid has $0.2 \mathrm{~m}$ intervals in both directions and the surface area of the measurement grid is $2 \times 2 \mathrm{~m}^{2}$. As in the simulations, the frequency of excitation is chosen to be $160 \mathrm{~Hz}$ (corresponding to $\lambda \simeq 2.15 \mathrm{~m}$ ). Note that this excitation frequency is in the frequency range where good attenuation is expected (see Fig. 10) and where the expected zone of quiet are not too small to be of practical use [2].

The change in sound pressure level measured around the target region in the case of a single sound source is shown in Fig. 11. For this control configuration, the average acoustic attenuation was $5.4 \mathrm{~dB}$ in the target region, as predicted by simulations (see Tab 1). Likewise, the shape and size of the quiet zone is very similar in both cases. As observed in the simulation the maximum increase in sound pressure level is in the near field of the secondary sources. The increase in SPL elsewhere is less than $4 \mathrm{~dB}$, except very close to the ground where it was about $8 \mathrm{~dB}$.

To further show the sensitivity of the optimal feedback controller to change in the primary disturbance, the control performance is investigated using multiple coherent primary sources. Not shown in this paper, seven additional loudspeakers were randomly distributed around the control system arrangement, at various height and distant by more than a wavelength from the control units. The change in sound pressure level obtained in the case of multiple coherent sound sources is shown in Fig. 12 , where an average acoustic attenuation of $4.4 \mathrm{~dB}$ was measured in the target region. Although the primary sound field is significantly changed from that produced by a single source, it can be observed that the feedback controller still allows decreasing the sound pressure level throughout the quiet zone. Note that except in the near field of the secondary sources and near the floor, the increase in sound pressure level means that the resulting sound field is more balanced. In case of multiple coherent sources, on the other hand, the contours of equal pressure reduction were considerably more complex due to constructive and destructive wave summation generated by the primary field in the room. It was observed that in regions where the primary noise level was initially low due to destructive interference, the sound pressure level was increased after control. Conversely, maximum acoustic attenuation was observed around areas where the primary noise level was initially high due to constructive interference.

\subsection{Discussion}

As shown above, the overall performance, shape and size of the experimentally produced quiet zone agree with the simulation results. The maximum attenuation is however slightly less, probably due to differences between the model and actual transducers and uncertainties in the pre-determined transfer paths. Moreover, it must be pointed out that the experimental conditions were not exactly the same as those considered in the simulation. In the computed results, a perfect matched layer was used to prevent sound reflection which may differ from the actual behaviour of the walls of the anechoic room. A sound hard boundary condition was also applied for the floor and the panel in the simulation. In the experiment, a half-inch thick wooden panel was used for both the floor (partially covered) and panel which could vibrate under the acoustic excitation. Despite these differences, an average sound reduction of about $6 \mathrm{~dB}$ is achieved in the target region through this control arrangement. From a practical point of view, the increase in the sound pressure level outside the quiet zone is found relatively low or located far from it (eg near the ground). It is also observed that the decentralised feedback controller still allows decreasing the 


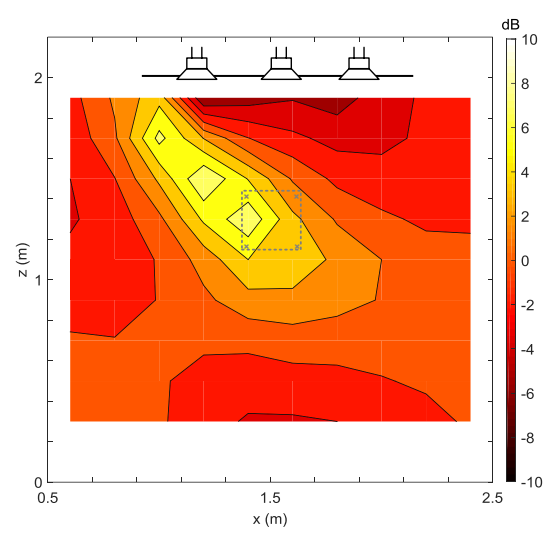

(a)

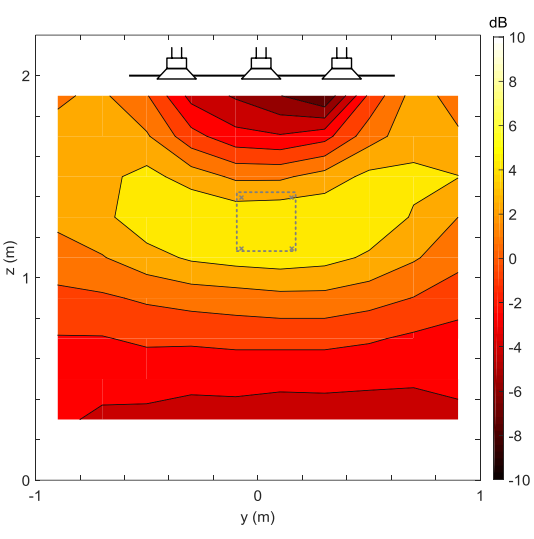

(b)

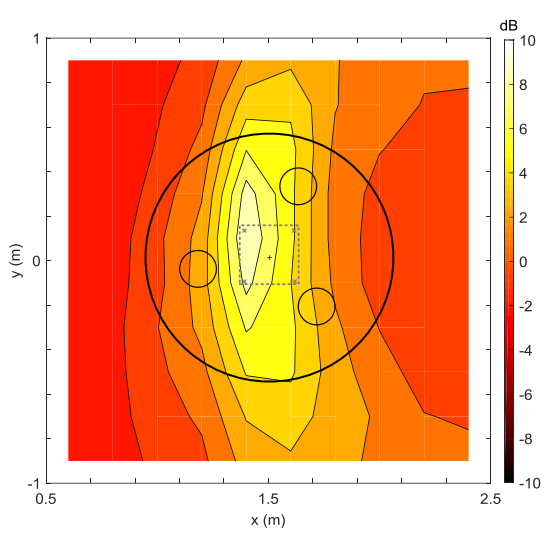

(c)

Figure 11: (color online) Change in sound pressure level measured in the case of a single primary source in the $x-z$ plane (a), in the $y-z$ plane (b), and the $x-y$ plane (c), respectively. The gray dotted square represents the target quiet area.

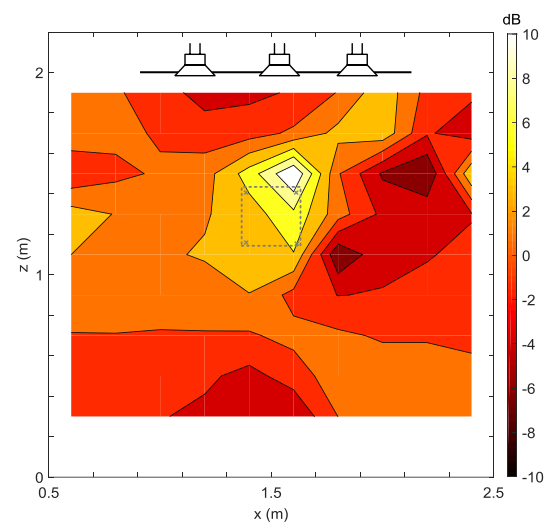

(a)

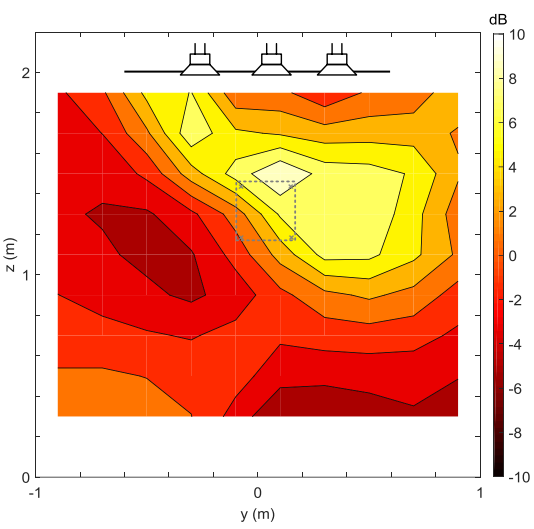

(b)

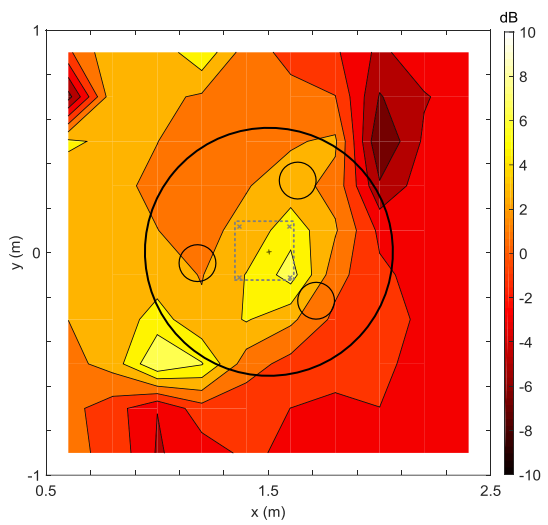

(c)

Figure 12: (color online) Change in sound pressure level measured with multiple coherent sound sources in the $x-z$ plane (a), in the $y-z$ plane (b), and in the $x-y$ plane (c), respectively. The gray dotted square represents the target quiet area.

sound pressure level around the target region even when the primary disturbance is generated by several additional sources randomly distributed in space. This further suggests that the proposed control strategy does not require highly accurate sound field prediction model. The method discussed in this paper opens up the possibility of designing such remote control systems in more complex enclosed sound fields.

A pure-tone sound field is considered in this paper, meaning that only the amplitude and phase of the feedback gains in Eq. (13) need to be determined. In many practical situations, however, the primary pressure field results from a large number of acoustic modes excited in an enclosure. If a broadband noise application is targeted, the challenging task will be the design of a stable and causal control filter that can be implemented in real time. Moreover, Fig. 10p shows the frequency response of the system studied in this paper between $30-300 \mathrm{~Hz}$, and Fig. 10 the expected performance as a function of the frequency. Although a broadband, diffuse primary field cannot be addressed yet, the complex envelope controller allows implementing any complex gains that can potentially be selected over a wide range of real parts and imaginary parts. This provides greater flexibility to achieve optimal acoustic attenuation. As is known, global control can be performed provided that a secondary source is distant by less than half a wavelength from the noise source [2. To make this research more meaningful and generalizable, the control units and primary source were spaced by about three half wavelengths. As discussed in previous work, the sound pressure level away from the control region can be further reduced by moving the minimization points near the secondary loudspeakers, but at the expense of a decrease of the size of the quiet zone 4. Some 
of the computed results presented in this study were obtained by taking into account an obstructing object in the target region. It is observed that placing a head-sized diffraction sphere in the middle of the quiet zone does not affect the performance of the control system. The presence of a rigid sphere affects the secondary sound field by imposing zero particle velocity, and thus zero pressure gradient. As has been shown by Garcia-Bonito and Elliott, this results in a beneficial flattening of the secondary field which tends to increase the spatial extent of the quiet zone close to the head 31. In addition, it has been found for the studied configuration that increasing the size of the diaphragm loudspeaker is beneficial to increase the size of the quiet zone, as already observed by Joseph et al. in the case of a single secondary loudspeaker and a single point in its near field.

\section{Conclusion}

It is shown in this study that a remote zone of quiet can be achieved using a control arrangement based on collocated loudspeaker-microphone pairs. A decentralised feedback control is considered since the cross-coupling between control units is weak, which may be useful when direct monitoring of the primary source can not be achieved. Another advantage is that the failure of one of the control unit is not likely to disrupt the stability of the system. Active noise reduction in the remote target region requires prior knowledge of the transfer path between the control units and a set of minimization points defining the desired quiet zone. The feedback gains are obtained by solving a linear quadratic optimization problem and implemented using a complex envelope controller, allowing any complex gain to be applied. An average noise reduction of about $6 \mathrm{~dB}$ was measured with a particular control arrangement in a target region of volume $0.25 \times 0.25 \times 0.25 \mathrm{~m}^{3}$ for a $160 \mathrm{~Hz}$ tonal primary source, distant more than one wavelength from the secondary sources. It has been found that the performance of this particular arrangement decreases when the primary source deviates by more than $50 \mathrm{deg}$ in azimuth from its initial position. Future research shall extend the concept to broadband, diffuse sound fields so that it is applicable to real world situations.

\section{Acknowledgement}

The authors would like to acknowledge the EPFL Metamedia Center for their financial support for this research.

\section{References}

[1] P.A. Nelson, A.R.D. Curtis, S.J. Elliott, A.J. Bullmore, The active minimization of harmonic en- closed sound fields, part I: Theory, Journal of Sound and Vibration 117 (1987) 1-13.

[2] P.A. Nelson and S.J. Elliott, Active control of sound, Academic Press, San Diego, CA, 1992.

[3] S.J. Elliott, P. Joseph, A.J. Bullmore and P. A. Nelson, Active cancellation at a point in a pure tone diffuse sound field, Journal of Sound and Vibration 120 (1988) 183-189.

[4] P. Joseph, E. J. Elliott and P. A. Nelson, Near field zone of quiet, Journal of Sound and Vibration 172 (1994) 605-627.

[5] A. David and S.J. Elliott, Numerical studies of actively generated quiet zones, Applied Acoustics 41 (1994) 63-79.

[6] S.J. Elliott and J. Garcia-Bonito, Active cancellation of pressure and pressure gradient in a diffuse sound field, Journal of Sound and Vibration 186 (1995) 696-704.

[7] J. Garcia-Bonito, S.J. Elliott, and C.C. Boucher, Generation of zones of quiet using a virtual microphone arrangement, The Journal of the Acoustical Society of America 101 (1997) 3498-3516.

[8] J. Guo, J. Pan, and C. Bao, Actively created quiet zones by multiple control sources in free space, The Journal of the Acoustical Society of America 101 (1997) 1492-1501.

[9] J. Guo and J. Pan, Further investigation on actively created quiet zones for multiple control sources in free space, The Journal of the Acoustical Society of America 102 (1997) 3050-3053.

[10] W.K. Tseng, Local active noise control using a novel method of designing quiet zones, Control Engineering Practice 19 (2011) 1450-1458.

[11] S.J. Elliott and T.J. Sutton, Performance of feedforward and feedback systems for active control, IEEE Transactions on Speech and Audio Processing 4 (1996) 214-223.

[12] B. Rafaely, S. J. Elliott, and J. Garcia-Bonito, Broadband performance of an active headset, The Journal of the Acoustical Society of America 106 (1999) 787-793.

[13] E. Leboucher, P. Micheau, A. Berry, and A. L'Espérance, A stability analysis of a decentralized adaptive feedback control system of sinusoidal sound in free space, The Journal of the Acoustical Society of America 111 (2002) 189-199.

[14] H.F. Olson, E.G. May, Electronic Sound Absorber, The Journal of the Acoustical Society of America, 25 (1953) 1130-1136. 
[15] H.F. Olson, Electronic control of noise, vibration and reverberation, The Journal of the Acoustical Society of America 28 (1956) 966-972.

[16] S.J. Elliott, P. Joseph, P.A. Nelson and M.E. Johnson, Power output minimization and power absorption in the active control of sound, The Journal of the Acoustical Society of America 90 (1991) 2501-2512.

[17] D. Guicking and E. Lorentz, An active sound absorber with porous plate, Journal of Vibration, Acoustics, Stress and Reliability in Design, 106 (1984) 389-392.

[18] M. Furstoss, D. Thenail, and M.A. Galland, Surface impedance control for sound absorption: direct and hybrid passive/active strategies, Journal of Sound and Vibration 203 (1997) 219-236.

[19] E. Rivet, S. Karkar, and H. Lissek, Broadband low-frequency electroacoustic absorbers through hybrid sensor-/shunt-based impedance control, IEEE Transactions on Control Systems Technology 25 (2017) 63-72.

[20] D. Moreau, B. Cazzolato, A. Zander and C. Petersen, A review of virtual sensing algorithms for active noise control, Algorithms, 1 (2008) 69-99.

[21] S. J. Elliott and A. David, A virtual microphone arrangement for local active sound control, 1st International Conference on Motion and Vibration Control, Yokohama, Japan, 1992.

[22] C. Kestell, B. S. Cazzolato, and C. H. Hansen, Active noise control in a free field with virtual sensor, The Journal of the Acoustical Society of America 109 (2001) 232-243.

[23] A. M. Finn, Active noise control system using phased-array sensors, US Patent number $5,699,437,(1997)$.

[24] S. Skogestad and I. Postletwaite, Multivariable feedback control, John Wiley \& Sons, Chichester (1996).

[25] S.J. Elliott, P. Gardonio, T.C. Sors and M.J. Brennan, Active vibroacoustic control with multiple local feedback loops, The Journal of the Acoustical Society of America 111(2) (2002) 908-915.

[26] J.Q. Sun, Some observations on physical duality and collocation of structural control sensors and actuators, Journal of Sound and Vibration 194(5) (1996) 765-770.

[27] D. J. Rossetti, M. R. Jolly, and S. C. Southward, Control effort weighting in feedforward adaptive control systems, The Journal of the Acoustical Society of America 99 (1996) 2955-2964.
[28] P. Micheau, R. Louviot and A. Berry, Decentralized resonant controller for vibroacoustic active control, Proceedings of the 15th Mediterranean Conference on Control and Automation, Athens, Greece, 2007.

[29] M. Michau, P. Micheau, R. Boulandet, A. Berry and P. Herzog, Decentralized complex envelope controller for ASAC by virtual mechanical impedances, IEEE/ASME International Conference on Advanced Intelligent Mechatronics, Besançon, France, 2014.

[30] N. Quaegebeur, P. Micheau and A. Berry, Decentralized harmonic control of sound radiation and transmission by a plate using a virtual impedance approach, The Journal of the Acoustical Society of America 125 (2009) 2978-2986.

[31] J. Garcia-Bonito and S. J. Elliott, Local active control of diffracted diffuse sound fields, The Journal of the Acoustical Society of America 98 (1995) 1017-1024. 